\title{
APSIDAL MOTION TEST: CONFRONTATION BETWEEN THEORY AND OBSERVATIONS
}

\section{A. CLARET ${ }^{\perp}$ AND A. GIMENEZ 1.2}

Instituto de Astrofísica de Andalucía, P.O.Box 3004, E-18080 Granada, Spain ${ }^{1}$ Laboratorio de Astrofísica Espacial y Física Fundamental, INTA P.O.Box 50727, E-28080 Madrid, Spain ${ }^{2}$

The apsidal motion test in eclipsing binaries is one of the few observational procedures available to study the stellar interior. A careful analysis of empirical data and theoretical models has been performed since more than ten years ago by our group, and in the present communication we show the most recent results obtained from the confrontation between theory and observations.

We have carried out an extensive comparison between new stellar models (Claret \& Giménez 1992) making use of the latest opacity library by Rogers \& Iglesias (1992) and the best available data from double-lined eclipsing binaries (Andersen, 1991). The level at which we can theoretically reproduce the observations of classical absolute dimensions, namely, $R$ and $T_{\text {eff }}$ for a given mass, is shown in Figure 1, while Figure 2 shows the fulfillment of the requirements of a common age for the two components of the same system. A very similar good agreement is obtained when we take into account a moderate amount of convective core overshooting. Small differences can be easily explained in terms of metal abundance variations with respect to the adopted average value $Z=0.02$. A more detailed check is shown in Figure 3 for some selected systems.

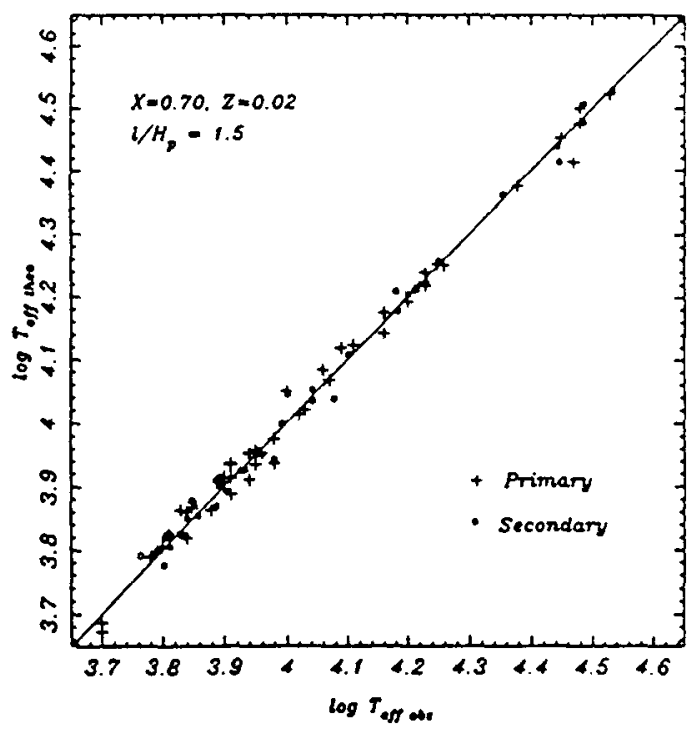

Fig. I Comparison between observed and theoretical effective temperatures. 


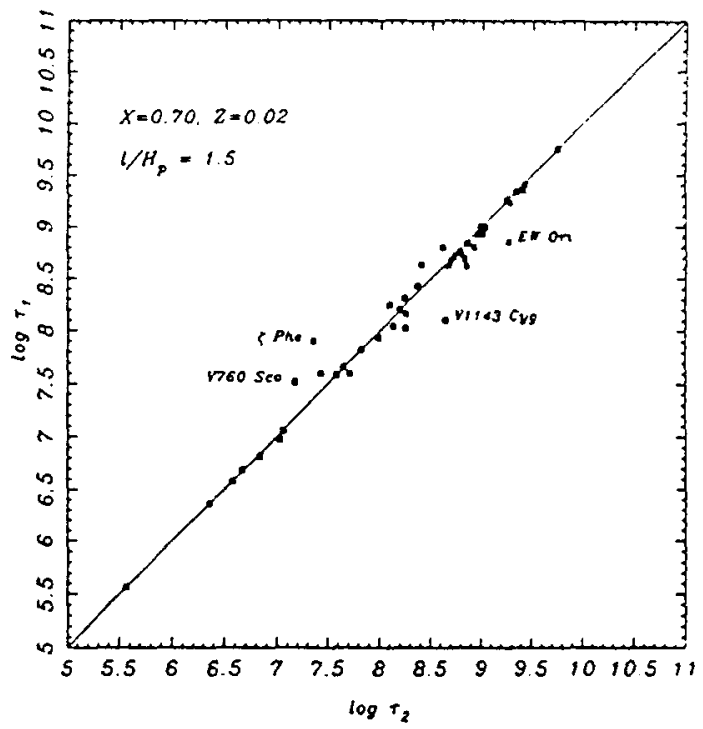

Fig. 2 Derived ages for individual components

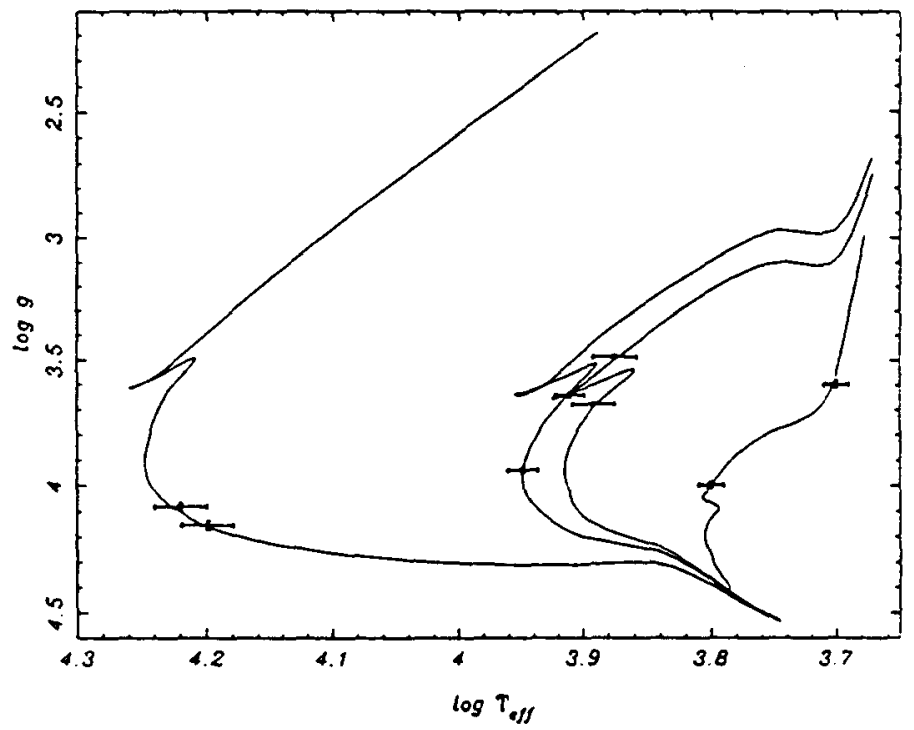

Fig. 3 Isochrones for some systems. From left to right: $U$ Oph $\left(5 \times 10^{7} \mathrm{y}\right)$, WX Cep $\left(6 \times 10^{8} \mathrm{y}\right), \mathrm{SZ}$ Cen $\left(7.9 \times 10^{8} \mathrm{y}\right)$ and AI Phe $\left(4.3 \times 10^{9} \mathrm{y}\right)$. For AI Phe, the corresponding isochrone was computed for $Z=0.01$. Note the differences in evolutionary status. 


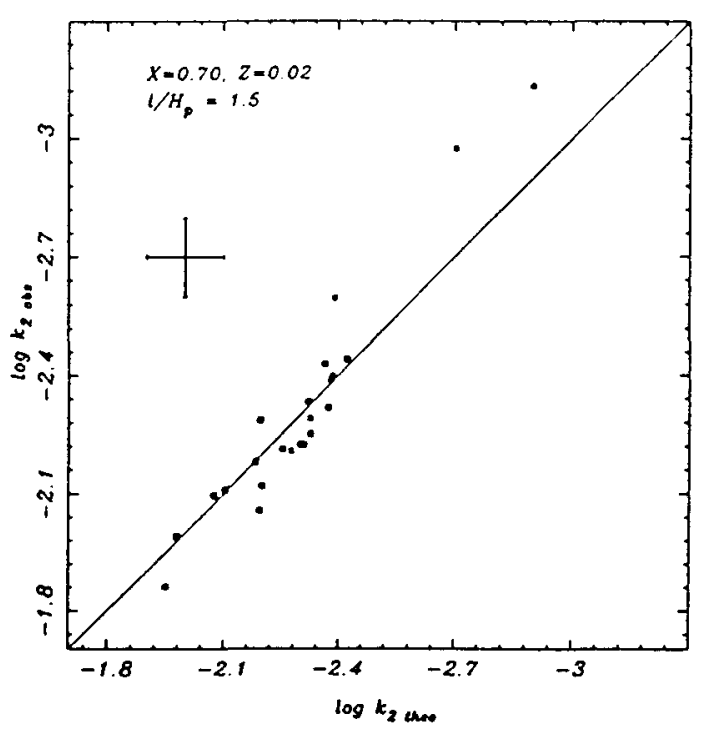

Fig. 4 Observed and predicted log $\mathbf{k}_{2}$. Systems with low quallity determination of absolute dimensions and/or apsidal motion rates are denoted by asterisks. Typical error bar is indicated.

Concerning the derived internal structure constant, $\log \mathbf{k}_{2}$, for the eccentric systems, we show in Figure 4 the comparison with theoretically predicted values using the same evolutionary models. The previously detected systematic differences between observations and models, in the sense that real stars appear to be more centrally concentrated in mass than theoretically predicted (Claret \& Giménez 1988; Giménez 1990), is for the first time not significantly measured. This result is obtained regardless of the assumption adopted of a moderate or none convective overshooting. Anyway, we have introduced stellar rotation in the evolutionary models which, concerning the apsidal motion constant, gives rise to more concentrated configurations in mass in good agreement with predictions by Stothers (1974).

We can conclude that the combination of absolute dimensions of the highest quality with accurately determined apsidal motion rates based on long term monitoring of eclipse timings, produces observational values of $\log k_{2}$ which are in reasonable good agreement with theoretical predictions when the latest available opacities and the most up-to-date input physics in general bave been taken into account.

\section{REFERENCES}

Andersen, J.: 1991, A\&AR, vol. 3, no. 2, 91

Claret, A., Giménez, A: 1988, in Astrophysics: Recent Progress and Future

Possibilities, Niels Bohr Institute, Copenhagen

Claret, A., Giménez, A: 1992, A\&AS, in press

Giménez, A: 1990, in Active Close Binaries, Kluwer Academic Publishers, ed. C.

Ibanoglu, pag. 137

Rogers, R. J., Iglesias, C. A.: 1992, ApJS, vol. 79, 507

Stothers, R.: 1974, ApJ, 194, 651 